\title{
Surgical Repair for Popliteal Venous Aneurysm Causing Severe or Recurrent Pulmonary Thromboembolism: Three Case Reports
}

\author{
Togo Norimatsu, MD, and Haruo Aramoto, MD, PhD
}

Poplitealvenous aneurysms (PVA) are associated with deep venous thrombosis and recurrentpulmonary thromboembolism (PE). We report three cases of PVA. In all three patients the first sign of PVA was acute PE; in one case, the PE was recurrent. Computed tomography and duplex ultrasonography revealed not only PE but also popliteal venous dilatation with thrombus. Surgical reconstruction was performed in each case after treatment for PE. No postoperative complications occurred, including recurrent PE. Surgical repair of PVA is safe and is a recommended treatment.

Keywords: popliteal venous aneurysm, pulmonary thromboembolism, surgical repair

\section{Introduction}

Venous aneurysms are uncommon, unlike varicose veins caused by valvular dysfunction. Popliteal venous aneurysm (PVA) is potentially life threatening, because it can result in pulmonary thromboembolism (PE). Because the reported risk of recurrent $\mathrm{PE}$ is high in patients with PVA, even among those receiving anticoagulation treatment, surgical intervention is recommended. ${ }^{1)}$ We report three cases of PVA treated with surgical reconstruction using different procedures.

\section{Case 1}

A 65-year-old woman presented with exertional dyspnea. Computed tomography (CT) revealed PE. Venous ultrasonography revealed a thrombosed multilocularsaccular PVA. There was no history of previous venous thrombosis. Anticoagulation therapy was started, and a temporary

Department of Vascular Surgery, Sakakibara Heart Institute, Tokyo, Japan

Received: September 22, 2014; Accepted: January 7, 2015 Corresponding author: Togo Norimatsu, MD. Department of Vascular Surgery, Sakakibara Heart Institute, 3-16-1 Asahi-cho, Fuchu, Tokyo 183-0003, Japan

Tel: +81-42-314-3111, Fax: +81-42-314-3133

E-mail: tonorima@shi.heart.or.jp inferior vena cava (IVC) filter (GüntherTulip; Cook Medical, Bloomington, Indiana, USA) was inserted. One day later, the patient became hypotensive and lost consciousness. Thrombosis of the pulmonary artery and popliteal vein were unchangedon repeat CT. The patient was intubated and was treated in intensive care for 27 days. Once her general condition stabilized, we performed surgical treatment for PVA. Lateral venorrhaphy was not possible because of inadequate healthy venous tissue. Aneurysmectomy and patch plasty with the saphenous vein were performed (Fig. 1).

\section{Case 2}

A 77-year-old woman presented with palpitations and a history of PE 2 years earlier. The patient's IVC filter had been removed, and she had discontinued oral anticoagulation therapy on her own. CT showed PE and a dilated right popliteal vein. Venous ultrasonography demonstrated a multilocularsaccular PVA that had grown over the preceding 2 years. We initiated anticoagulation therapy after IVC filter placement. The patient underwent aneurysm resection with interposed polytetrafluoroethyleneprosthesis (PTFE) 17 days later (Fig. 2).

\section{Case 3}

A 76-year-old woman presented to another hospital with acute shortness of breath. She was admitted and treated there for heart failure. Two weeks later, the patient was transferred to our hospital because of hypoxia and hypotension. CT showed PE and deep vein thrombosis. Ultrasonography revealed right PVA and thrombus. A temporary IVC filter (ALN vena cava filter; ALN, Ghisonaccia, France) was placed and tissue-type plasminogen activator was administered, after which the patient's condition markedly improved. The patient electively underwent tangential aneurysmectomy and lateral venorrhaphy (Fig. 3).

The pathologic findings of the excised tissues were inconsistent with varicose veins. At 21-57-month follow-up, 

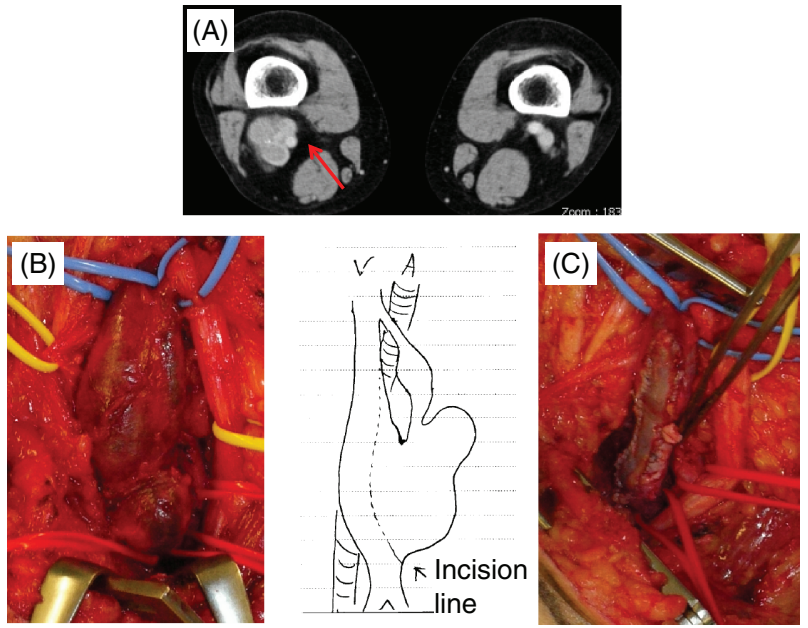

Fig. 1 Case 1. (A) Computed tomography (CT) scan showing multilocular saccular popliteal venous aneurysm (PVA; arrow). (B) Intraoperative view and schema of PVA. (C) Aneurysmectomy and patch plasty with saphenous vein.
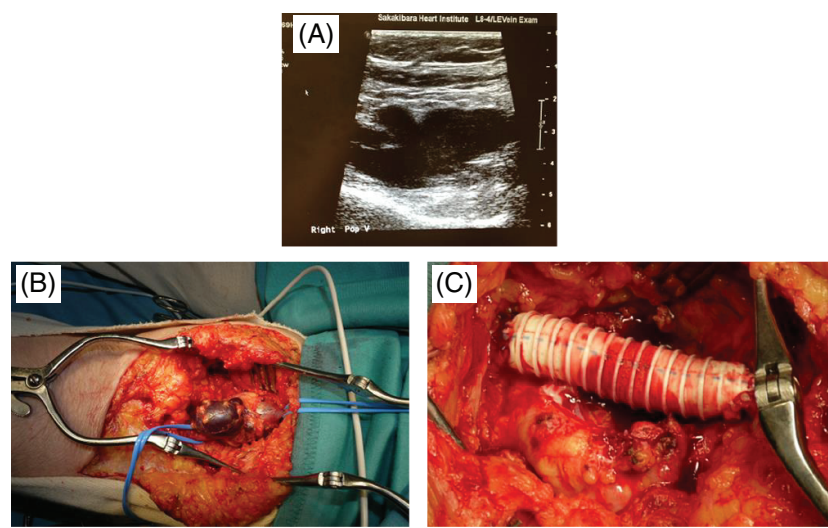

Fig. 2 Case 2. (A) Duplex scan of multilocular saccular PVA. (B) Intraoperative view of PVA. (C) Aneurysm resection with interposed polytetrafluoroethylenegraft. PVA: popliteal venous aneurysm.

duplex scanning demonstrated deep venous system patency without any symptoms in all cases.

\section{Discussion}

PVA is rare. There have been 212 reported cases since the first report by May and Nisselin 1968.,2,3) In 1976, Dahl et al. described the first case of recurrent PE resulting from PVA. ${ }^{4)}$ According to a current review, $24-51 \%$ of patients with PVA present with PE. In $55-76 \%$ of patients, PVA is associated with chronic venous disease, including superficial vein insufficiency, leg swelling, and venous ulceration. ${ }^{1,5)}$ Rupture is a rare complication of PVA. ${ }^{5}$ The etiology of PVA remains unknown, and no association with arterial aneurysm has been reported.

Various modalities are available to diagnose PVA, including phlebography, duplex ultrasonography, and computed

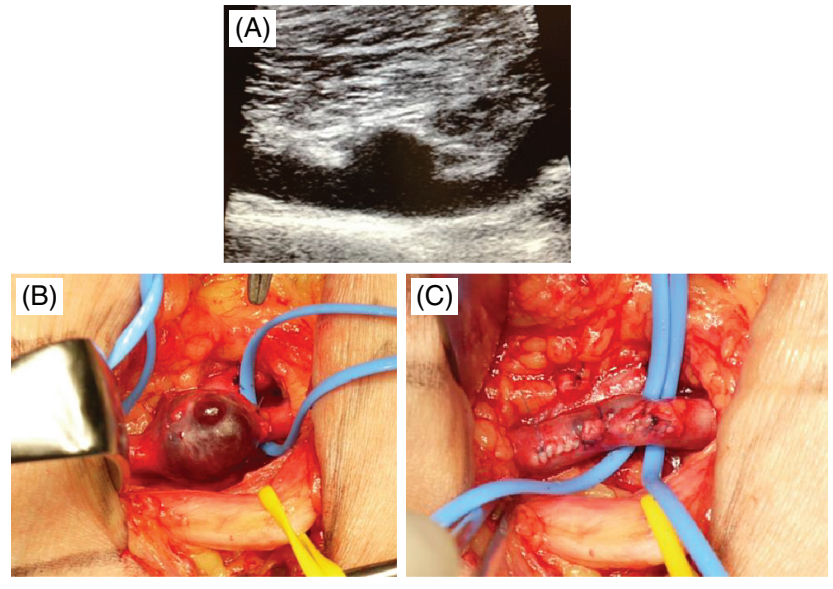

Fig. 3 Case 3. (A) Duplex scan of simple saccular PVA. (B) Intraoperative view of PVA. (C) Tangential aneurysmectomy and lateral venorrhaphy. PVA: popliteal venous aneurysm.

tomography (CT). Venous duplex scanning is the best noninvasive diagnostic method to assess lower limb deep vein aneurysm, and to determine aneurysm size and morphology.

Most PVAs are saccular (72-88\% of cases); the remainder are fusiform. ${ }^{1,6)}$ Thrombus formation within the aneurysmal sac is found in approximately two-thirds of PVA patents. Although large or saccular aneurysms are more prone to thromboembolic complications, there are no size criteria to definitely label a venous fusiform dilatation of an aneurysm. Maleti et al. defined aneurysm as a venous fusiform dilatation $>20 \mathrm{~mm}$, at least three times the size of the normal popliteal vein.7)

Anticoagulation therapy alone may not prevent PE in patients with symptomatic PVA, and its sole use as treatment is associated with a high incidence of recurrence. . $^{1,3,5,6,8)}$ Given the potential for serious thromboembolic complications, surgical repair is indicated in all symptomatic patients. However, management of asymptomatic PVA remains a controversial issue.

Two case series have described the course of asymptomatic patients with fusiform and saccular PVAs. ${ }^{1)}$ Although untreated, none of these patients experienced thromboembolic events. However, the size of the aneurysms was quite small and follow-up was short. Maldonado-Fernandez et al. reported five deaths resulting from $\mathrm{PE}$ in patients receiving medical treatment for PVA. ${ }^{3)}$ Therefore, regardless of symptoms, most investigators consider surgery is the best treatment for PVA, indicated for all saccular aneurysms and for fusiform aneurysms $>20 \mathrm{~mm}$.

PVA does not present with specific, definite signs, or symptoms. Only $20 \%$ of reported cases had a palpable mass in the popliteal fossa, ${ }^{3)}$ making PVA difficult to diagnose in asymptomatic patients. In these cases, there is no need to pursue duplex ultrasonography as a screening 
test. However, all PE patients should undergo CT phlebography and duplex ultrasonography with PVA in mind. As shown in our three cases, PE with PVA can be severe or recurrent, and a cure is possible with surgery.

Maldonado-Fernandez et al. reviewed the records of 102 patients who underwent surgical treatment for PVA. ${ }^{3)}$ No mortality, major complications, or repeat thromboembolic events occurred in these patients. However, there were several minor complications. Early complications, including hematoma, transient nerve injury, infection, and thrombosis of the surgical repair, occurred in $20 \%$ of patients. Late complications developed in $4 \%$, including thrombosis from the procedure (one case) and relapse of the venous aneurysm (three cases).

Various operative procedures have been used for venous reconstruction and the incidence of complications varies greatly according to operation method. Aneurysm resection with preservation of venous continuity is recommended. In particular, tangential aneurysmectomy with lateral venorrhaphyis the preferred technique, because of its lower complication rate compared with other procedures. In contrast, resection of aneurysms with end-toend anastomosis is not considered effective because it carries a high risk of early thrombosis.

Only one of our cases was treated with tangential aneurysmectomy with lateral venorrhaphy. Direct suturing was not possible in the other cases because of inadequate remaining healthy venous area or large native venous diameter. In case 2 , the native venous diameter after PVA resection was $10 \mathrm{~mm}$, making it unsuitable for interposition with a saphenous veingraft. Generally, reconstruction using an autologous graft is the preferred method. However, thrombosis has been reported in a patient who received an interposition graft using the internal jugular vein. ${ }^{6}$ Although we considered using a spliced vein graft, we opted for a ringed PTFE graft, which has high antithrombogenicity, is not difficult to use, and is not timeconsuming. IVC filter placement is not recommended in current American College of Chest Physicians or European Society of Cardiology guidelines on PE treatment. Patient 1 deteriorated substantially despite IVC filter implantation. We could not determine whether new embolization had occured through the filter or pulmonary embolism had progressed. However, preventive IVC filter placement is thought to reduce the risk of embolism during surgical repair of deep vein aneurysm or when thrombosis recurs in the surgical area. All of our patients had acute PE with PVA on admission. The IVC filter was placed to allow subsequent surgery. In case 3 , the thrombus disappeared within 10 days, and the patient did not wish to pursue surgery immediately. Hence, the IVC filter was removed, and surgery was performed approximately 5 months later. Most patients receive oral anticoagulation for 3 to 6 months. ${ }^{1,3,9)}$ However, we recommended permanent use of compression stockings and oral anticoagulationtherapy with Vitamin K antagonist to our patients, because all three had severe PE.

\section{Conclusion}

PVA is uncommon but can cause fatal or recurrent PE. Therefore, PVA should be kept in mind in cases of thromboembolism. The most effective treatment to prevent PE in patients with PVA is surgical repair rather than anticoagulation therapy.

\section{Disclosure Statement}

The authors have no conflicts of interest to report.

\section{References}

1) Sessa C, Nicolini P, Perrin M, et al. Management of symptomatic and asymptomatic popliteal venous aneurysms: a retrospective analysis of 25 patients and review of the literature. JVasc Surg 2000; 32: 902-12.

2) May R, Nissel R. Aneurysma der Vena poplitea. Rofo Fortschr Geb Rontgenstr Neuen Bildgeb Verfahr 1968; 108: 402-3. (in German)

3) Maldonado-Fernandez N, Lopez-Espada C, MartinezGamez FJ, et al. Popliteal venous aneurysms: results of surgical treatment. Ann Vasc Surg 2013; 27: 501-9.

4) Dahl JR, Freed TA, Burke MF. Popliteal vein aneurysm with recurrent pulmonary thromboemboli. JAMA 1976; 236: 2531-2.

5) Gabrielli R, Rosati MS, Siani A, et al. Management of symptomatic venous aneurysm. Scientific World J. 2012; Article ID 386478, 6 pages.

6) Aldridge SC, Comerota AJ, Katz ML, et al. Popliteal venous aneurysm: report of two cases and review of the world literature. J Vasc Surg 1993; 18: 708-15.

7) Maleti O, Lugli M, Collura M. Anévrysmes veineux poplites: expérience personnelle. Phlébologie. 1997; 50: 53-9. (in French)

8) Gabrielli R, Vitale S, Constanzo A, et al. Our experience of popliteal vein aneurysm. Interact Cardiovasc Thorac Surg 2010; 11: 835-7.

9) Gasparis AP, Awadallah M, Meisner RJ, et al. Recurrent popliteal vein aneurysm. J Vasc Surg 2010; 51: 453-7. 